\title{
Does locally delivered Zoledronate influence peri-implant bone formation? - Spatio-temporal monitoring of bone remodeling in vivo
}

\author{
Ulrike Kettenberger $^{\mathrm{a}}$, Julien Ston ${ }^{\mathrm{a}}$, Eric Thein ${ }^{\mathrm{b}}$, Philip Procter ${ }^{\mathrm{c}}$, Dominique P. Pioletti ${ }^{\mathrm{a}}{ }^{*}$ \\ a Laboratory of Biomechanical Orthopedics, Institute of Bioengineering, École Polytechnique Fédérale de Lausanne (EPFL), Switzerland \\ b Orthopaedic and Traumatology Department, University Hospital Lausanne (CHUV), Switzerland

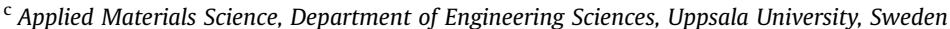

\section{A R T I C L E I N F O}

Article history:

Received 16 July 2014

Accepted 1 September 2014

Available online 18 September 2014

\section{Keywords:}

Drug delivery

Hydrogel

Bisphosphonate

Implant

Dynamic histomorphometry

MicroCT

\begin{abstract}
A B S T R A C T
Bisphosphonates are known for their strong inhibitory effect on bone resorption. Their influence on bone formation however is less clear. In this study we investigated the spatio-temporal effect of locally delivered Zoledronate on peri-implant bone formation and resorption in an ovariectomized rat femoral model. A cross-linked hyaluronic acid hydrogel was loaded with the drug and applied bilaterally in predrilled holes before inserting polymer screws. Static and dynamic bone parameters were analyzed based on in vivo microCT scans performed first weekly and then biweekly. The results showed that the locally released Zoledronate boosted bone formation rate up to $100 \%$ during the first 17 days after implantation and reduced the bone resorption rate up to $1000 \%$ later on. This shift in bone remodeling resulted in an increase in bone volume fraction (BV/TV) by $300 \%$ close to the screw and $100 \%$ further away. The double effect on bone formation and resorption indicates a great potential of Zoledronateloaded hydrogel for enhancement of peri-implant bone volume which is directly linked to improved implant fixation.
\end{abstract}

๑) 2014 Elsevier Ltd. All rights reserved.

\section{Introduction}

The human skeleton is constantly renewed and repaired by the bone remodeling process, a well-coordinated sequence of osteoclast-mediated bone resorption and osteoblast-mediated bone formation [1,2]. Anti-catabolic drugs, such as bisphosphonates (BPs), can slow down this process and shift the balance between bone formation and resorption activity by selectively affecting osteoclast function [3]. Osteoclasts are considered to be the main target for BPs as only this cell type can liberate and internalize mineral bound BPs during the resorption process [4,5]. However, effects of BPs on cells of the osteoblast lineage have also been described, which raises the question if BPs can also enhance bone formation activity [6,7]. In vitro experiments have shown that low concentrations of BPs can protect osteoblasts and osteocytes against apoptosis [8,9], stimulate mineralized bone nodule formation [10], and have a beneficial effect on osteoblast differentiation and protein synthesis [11]. Specifically for Zoledronate, the most potent bisphosphonate used in clinics today, an improved

\footnotetext{
* Corresponding author. EPFL, STI, IBI, LBO, Station 19, 1015 Lausanne, Switzerland. Tel: +41 21693 8341; fax: +41 216938660 .

E-mail address: dominique.pioletti@epfl.ch (D.P. Pioletti).
}

mineralization and proliferation of human osteoblast-like cells has been demonstrated [12]. However, conflicting results can also be found showing that BPs might inhibit mineralization and osteoblast growth, induce osteoblast apoptosis, and inhibit protein prenylation in osteoblasts in a dose-dependent manner [7,13]. Taken together, these findings suggest that BPs might, in addition to their known anti-resorptive effect, also have the ability to stimulate bone formation.

BPs are usually administered orally or intravenously. Local application is of particular interest in cases where existing bone needs to be preserved and augmented whilst avoiding the known side effects related to systemic administration [14,15]. Several in vivo studies have shown that a local delivery of BPs can enhance peri-implant bone density [16,17], implant fixation [18-22], and the repair of bony defects $[23,24]$ in animal models and humans. Furthermore BPs have been shown to preserve bone allografts $[25,26]$ as well as bone sites affected by osteonecrosis [27].

Currently published studies are mostly based on static analysis techniques like single $\mathrm{CT} /$ microCT scans, histology or pull-out/ push-out tests performed after euthanasia of the animals $[18,22,28]$. These techniques do not allow a separate investigation of the dynamic bone formation and bone resorption processes. Few studies can be found that looked at dynamic bone parameters after a systemic administration of BPs, but they did not show enhanced 
bone formation activity in humans nor in animals [29,30]. Some studies even demonstrated reduced bone formation and a suppressed activity of pre-existing bone-forming surfaces at medium to high doses of BPs [31-35].

Therefore, the aim of the present study is to investigate the spatio-temporal effect of locally delivered Zoledronate on periimplant bone remodeling using the so-called dynamic histomorphometry. This previously developed microCT-based technique enables us to quantitatively measure the average outcome of bone formation and resorption over long time periods [36,37], allowing an investigation of the so far unclear effect of locally applied BPs on the dynamic bone formation processes. Understanding the effects of Zoledronate and how it interacts with normal bone healing is important when it comes to designing drug delivering implants and materials. The active range, the time delay, and duration of the BPs' effects (very local versus widespread, fast versus slow, long term versus short-term effects) are crucial for targeting specific bone sites, as well as certain phases of bone healing.

\section{Materials and methods}

\subsection{General study design}

Custom-made polymeric miniature screws were implanted bilaterally in the femoral condyles of ovariectomized (OVX) rats. Unlike metal implants, these screws are visible on microCT scans without creating image disturbing artifacts. A hydrogel based drug delivery system was used to release Zoledronate into the peri-implant bone stock. During surgery, the hydrogel was inserted into the predrilled screwhole before screw implantation. The study included 3 experimental groups with 4 animals assigned to each group. In the first group, the hydrogel was loaded with $5 \mu \mathrm{g}$ Zoledronate (Zol-Gel-group); in the second group, unloaded hydrogel was used (Gel-group); and in the third group, no hydrogel was inserted (Control-group) before screw implantation. Time-lapsed in vivo microCT scans of the animals were performed for close monitoring of the bone response to the implantation and how it is altered by the bisphosphonates delivery. A time line of the study is shown in Fig. 1.

\subsection{Hydrogel preparation}

A commercially available cross-linked hyaluronic acid (Termira AuxiGel ${ }^{\mathrm{TM}}$, Stockholm, Sweden) was used as the drug delivery system for the BPs because this gel had already been shown to be a suitable material for delivering drugs such as growth factors to bone [38-41]. The crosslinking of the hydrogel is achieved by mixing a hyaluronan derivative (component A) with a PVA cross-linker (component B). First, an aqueous solution containing $2 \mathrm{mg} / \mathrm{ml}$ Zoledronate (Art.-Nr. ALX-430153-0000, Enzo Life Sciences, Farmingdale, USA) was prepared. The exact drug dose was ensured by mixing precisely weighed and measured amounts of Zoledronate and distilled water. All solutions were sterile filtered and the mixing of the gels was performed under sterile conditions. Five parts of the Zoledronate solution were mixed with 3 parts of component A and 2 parts of component B for the Zol-Gelgroup. The Zoledronate solution was replaced by bi-distilled water for the Gel-group. The resulting gels were allowed to settle for $1 \mathrm{~h}$ before filling the capillary pistons of a positive displacement pipette (Microman ${ }^{\circledR}$, Gilson, Middleton, USA) with $5 \mu$ l gel each. The capillaries were pulled off the pipette with the gel inside and left $1 \mathrm{~h}$ for settling. Then they were sterile packed in plastic tubes and frozen at $-20{ }^{\circ} \mathrm{C}$.

\subsection{Animal model}

All animal procedures were approved by the local animal care and use committee (license no. 2508.1, EXPANIM, SCAV, Epalinges, Switzerland). Twelve 14 week old virgin female rats were received from Janvier Labs (Saint-Berthevin, France) and acclimatized in the animal facilities for 3 weeks. All rats were housed 4 per cage under $12 \mathrm{~h}$ light to $12 \mathrm{~h}$ dark cycles at $22{ }^{\circ} \mathrm{C}$ room temperature with $55 \%$ humidity. They were fed with a standard rodent diet (KLIBA NAFAG 3436, Provimi Kliba AG, Switzerland) and tap water at libitum. After ovariectomy (OVX), food intake was limited to $50 \mathrm{~g}$ per kg body weight per day. Sterilized hay, paper tunnels and wooden sticks were offered as cage environment enrichment. The animals were fed in groups and equal nutrition of all animals was ensured by close monitoring and regular weighing.

\subsection{Surgical procedures}

\subsubsection{Ovariectomy}

The rats were ovariectomized bilaterally at an age of 17 weeks and a weight of $293 \pm 11 \mathrm{~g}$ to induce an estrogen deficiency related bone loss [42]. We used a dorsal approach similar to the procedure described by Alghamdi et al. [43]. Buprenorphine (Temgesic $^{\circledR}$, Reckitt Benckiser AG, Wallisellen, Switzerland) was administered subcutaneously before surgery and every $8 \mathrm{~h}$ for the first $48 \mathrm{~h}$ after surgery for pain relief. Paracetamol (Dafalgan 500 mg effervescent tablet, UPSA Bristol-Myers Squibb SA, Baar, Switzerland) was added to the drinking water of the rats for 5 days. The skin incisions were closed with running subcuticular sutures (Vinyl 5.0, Ethicon, Somerville NJ, USA) so that the rats did not have to be kept in separate cages after the surgery. No antibiotics were given.

\subsubsection{Screw implantation}

Screws were implanted when the rats were aged 22 weeks and weighed $338+18$ g. One miniature screw was implanted in each femoral condyle under isoflurane anesthesia with Buprenorphine to avoid intraoperative pain. The surgery was done under aseptic conditions. The rat was prepared for surgery and the first leg was fixed on a special table with the knee in a flexed position. A skin incision of $1-2 \mathrm{~cm}$ was made on the lateral side of the distal femoral end and the muscles were blunt dissected in order to expose the condyle. One hole with a diameter of $1.2 \mathrm{~mm}$ and $3.5 \mathrm{~mm}$ length was drilled unicortically in the condyle with a motorized dentist's drill (DEC 100, Nobelcare, Sweden). The cortex was pretapped to avoid deformation and damage to the polymer screws. The drilled hole was rinsed with saline solution and surgical vacuum was used to remove bone particles. As a next step, the hydrogel was inserted in the predrilled hole with a positive displacement pipette before implanting a miniature screw (Fig. 2). The screws with a thread length of $3 \mathrm{~mm}$ and a diameter of $1.4 \mathrm{~mm}$ were custom made from radiopaque polyetheretherketone (RISystem, Davos, Switzerland) with a $100 \mathrm{~nm}$ titanium coating to mimic the interface with a standard orthopedic screw. After implanting the screw, the fascia of the muscle was closed with resorbable sutures (Vinyl 5.0). The skin was closed with interrupted subcuticular sutures (Vicryl 5.0) before performing the same procedure at the contralateral femur. The rat was placed in a heated incubator for post-surgical recovery. The post-OP medication and care was similar to the procedure after ovariectomy described above.

\subsection{MicroCT imaging, data processing and analysis}

\subsubsection{In vivo microCT imaging}

In vivo microCT scans (Skyscan 1076, Bruker microCT, Kontich, Belgium) of only the right femur were performed one day before OVX and one day before screw implantation in order to confirm the bone loss caused by the estrogen deficiency. Both femurs were then scanned at days 3, 10, 17, 31, 45, and 58 after screw implantation for the dynamic histomorphometry. According to literature, no bone damage due to radiation can be expected after 8 consecutive microCT scans [44]. The animals were kept under isoflurane anesthesia during the scans to avoid motion artifacts. The rats were euthanized at the time of the last microCT scan with an intraperitoneal injection of pentobarbital (Esconarkon, Streuli Pharma SA, Uznach) while under anesthesia.

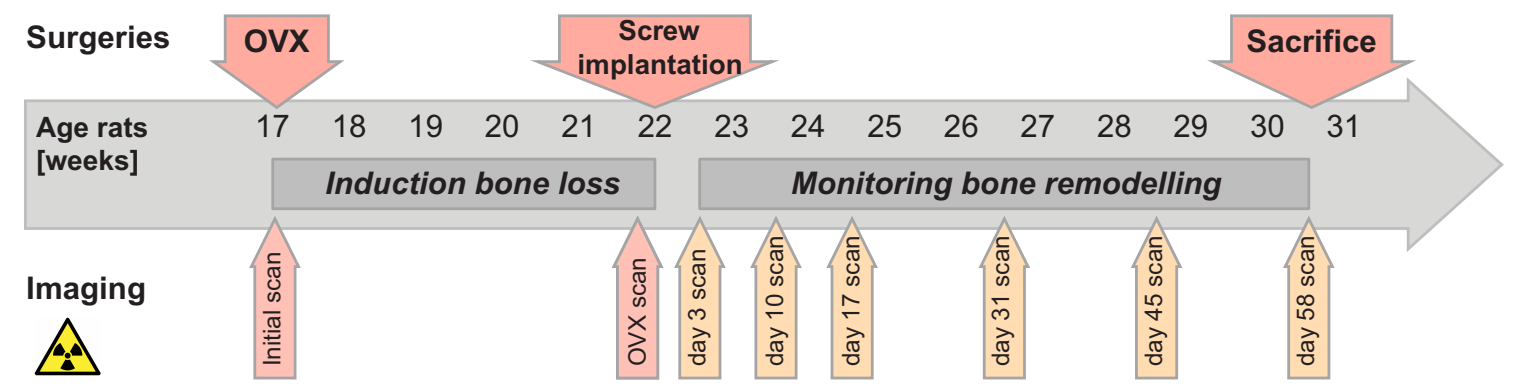

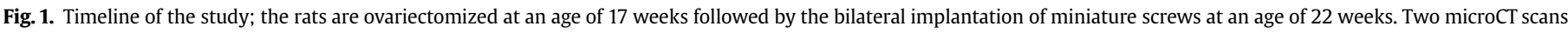
were performed to confirm the bone loss after OVX, 6 more were performed after the screw implantation to monitor bone remodeling. 


\section{Drilling}

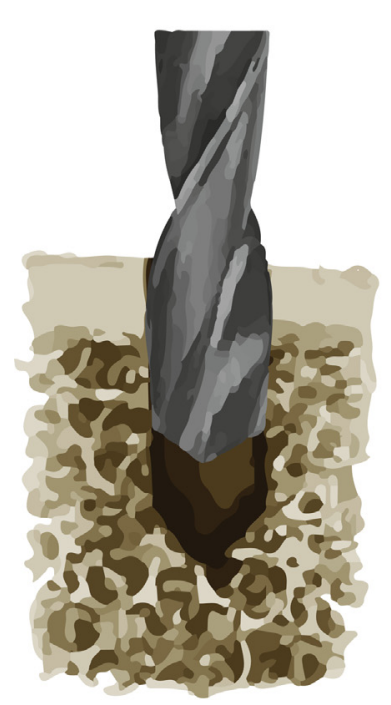

2. Hydrogel insertion

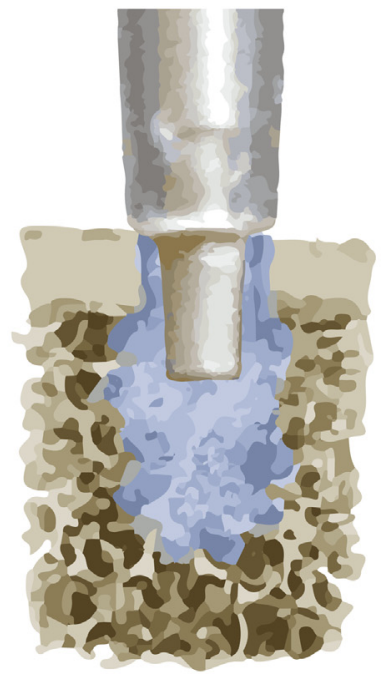

3. Screw implantation

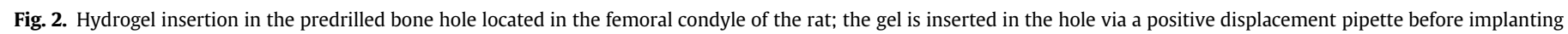
the screw.

\subsubsection{Data acquisition and reconstruction}

The parameters for the scans assessing the bone loss before screw implantation were the following: $0.5 \mathrm{~mm}$ aluminum filter, voltage $60 \mathrm{kV}$, current $167 \mu \mathrm{A}$, exposure time $480 \mathrm{~ms}$, rotation step $0.4^{\circ}$. The peri-implant bone after screw insertion was scanned with different parameters adapted to the density of the screw: $0.5 \mathrm{~mm}$ aluminum filter, voltage $80 \mathrm{kV}$, current $120 \mu \mathrm{A}$, exposure time $400 \mathrm{~ms}$, rotation step $0.5^{\circ}$. The spatial resolution of all scans was $18.4 \mu \mathrm{m}$. Two polymer-hydroxyapatite phantoms with known mineral density were scanned under the same conditions as the rats and served for a calibration of the bone mineral density (BMD) and tissue mineral density (TMD) measurement histograms. The reconstruction of the projection images was done with NRecon and GPURecon Server (Bruker microCT, Kontich, Belgium). A ring artifact correction of 4 and a beam hardening correction of $20 \%$ for the scans assessing bone loss and $30 \%$ for the peri-implant bone scans were set to improve the image quality. The reconstruction output was stacks of crosssections in 8-bit bitmap format.

\subsubsection{Assessment of the estrogen deficiency induced bone loss}

The scans taken before OVX and before screw implantation were analyzed with the software CTan (Bruker microCT) to confirm the induced bone loss. A volume of interest (VOI) with a length of $4 \mathrm{~mm}$ was defined proximal to the growth plate located in the femoral condyle. Cortical and trabecular regions were selected automatically with a customized algorithm before analyzing the morphometric parameters as well as the BMD of the trabecular region and the TMD of the cortical region.

\subsubsection{MicroCT-based dynamic histomorphometry}

The microCT datasets were used to perform dynamic histomorphometry, a technique that is based on the registration and comparison of two consecutive
microCT scans $[36,37]$. Bone was considered to be resorbed if it was present on the first microCT scan and not on the second, whereas bone was considered to be formed if it was present on the second scan and did not appear on the first. Bone which appeared in both scans was considered to be quiescent. In this way, bone remodeling can be monitored in three dimensions over long periods of time without the need to euthanize the animals.

Image processing for the dynamic histomorphometry was done with Amira ${ }^{\circledR}$ (FEI Visualization Sciences Group, Burlington, USA) and CTan. In the femoral condyle, a VOI of $4.5 \mathrm{~mm}$ in height around the screw was manually defined with CTan for all animals. Using an automated algorithm, the background and the patella were removed and the dataset size was reduced to facilitate image processing. Then all datasets were loaded into Amira ${ }^{\mathbb{B}}$ for further processing with a custom script. The script first super positioned all original gray value images using the built-in registration function and their correlations as a similarity measure. Each dataset was registered with the image from the previous time step. All images were transformed into the coordinate system of the first image using the built-in standard interpolation method and the datasets were filtered with a median-based 3D noise-reduction filter. Next, a VOI containing only trabecular bone was automatically defined on the first dataset and adapted manually so that it would also fit consecutive scans which are slightly different due to ongoing bone remodeling. Then, for all datasets, the VOI was binarized with a threshold of 80 . By comparing consecutive scans voxel by voxel, resorbed, formed, and quiescent bone regions were identified and assigned one of three gray values. Two additional gray values were assigned to the screw and the bone marrow. These images were saved and loaded into CTan. The static and dynamic bone parameters around the screw were measured in four layers of $368 \mu \mathrm{m}$ (20 voxels) (Fig. 3). The static bone parameters bone volume fraction (BV/TV), trabecular thickness (Tb.Th), trabecular number (Tb.N), trabecular separation (Tb.Sp), and structure model index (SMI) were analyzed for a complete assessment

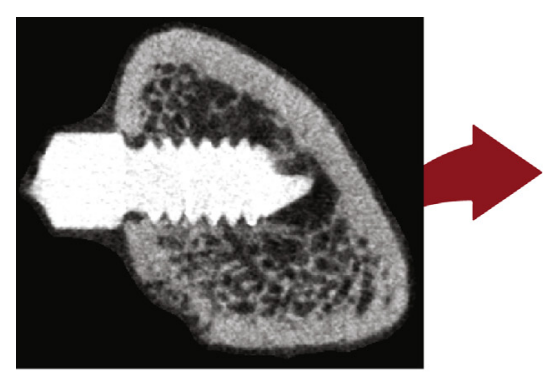

Original $\mu \mathrm{CT}$ dataset background removed

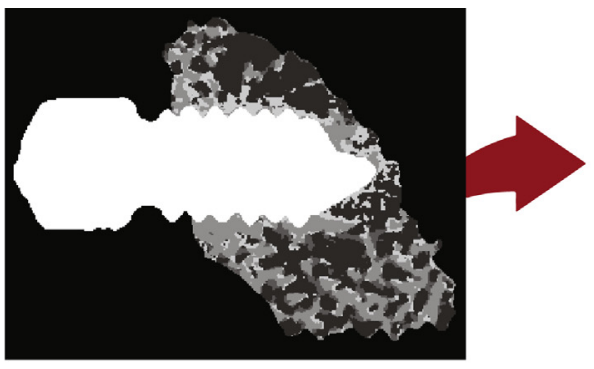

Comparison 2 time points cortex removed

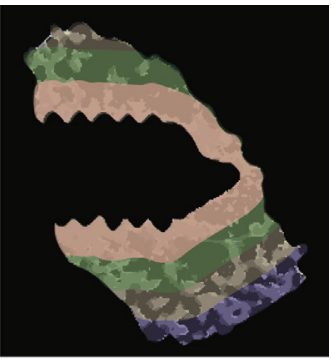

$368 \mu \mathrm{m}$ layers for analysis

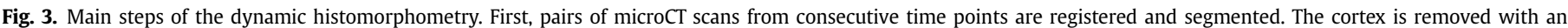

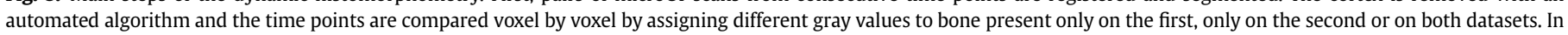
a last step, 4 layered VOIs of $368 \mu \mathrm{m}$ each are created and analyzed separately for static and dynamic bone parameters. 
of the bone structure changes. The volume-based bone formation rate (BFR) and bone resorption rate (BRR) were chosen as representative parameters as their calculation is robust and not as sensitive to registration inaccuracies as the surfacebased parameters. The bone formation rate is given in percent per day (\%/d) and calculated as the ratio of formed bone volume to quiescent bone volume divided by the number of days between the two scans. The bone resorption rate is calculated as the ratio of resorbed bone volume to quiescent bone volume divided by the days between the scans.

\subsection{Histology}

The rat femurs were dissected just after euthanasia and fixed in $4 \%$ paraformaldehyde solution before dehydrating them in a series of ethanol solutions with ascending concentration (70\%, 80\%, 90\%, 95\% and 100\%). After clearing the samples in toluol, they were embedded by infiltration with methylmethacrylate $99 \%$ (MMA), bis(tert-butylcyclohexyl) peroxydicarbonate (Perkadox 16) and dibutylphtalate (DBP). All agents were purchased from Sigma-Aldrich (St. Louis, US). After polymerization during 3 weeks at room temperature, the samples were cut with a diamond-coated inner diameter saw (Leica SP 1600, Leica Microsystems, Wetzlar, Germany) to slices of around $200 \mu \mathrm{m}$ thickness. Those slices were glued with acrylic glue (Loctite 401, Henkel, Düsseldorf, Germany) to custom-made opaque PMMA microscope slides (Semadeni, Ostermundigen, Switzerland) and ground to around $60 \mu \mathrm{m}$ thickness with a grinding machine (Pedemax-2, Struers, Willich, Germany). Finally, the surface of the slides was etched with $0.7 \%$ formic acid (Applichem, Gatersleben, Germany) before staining it with toluidine blue (VWR, Dietikon, Switzerland). Images were taken with an upright light microscope (DM 5500, Leica Microsystems, Wetzlar, Germany).

\subsection{Statistics}

Statistical testing was done with Matlab (Mathworks, Natick, MA, USA). Values lying outside an interval of 1.5 times the quartile range were identified as outliers and excluded. The results of the OVX scans were analyzed with a paired $t$-test after performing a Lilliefors test for a normal distribution. In case the normal distribution could not be confirmed, a Wilcoxon singed rank test was used. The static and dynamic bone parameters were tested for significance with a non-parametric Kruskal Wallis ANOVA followed by a Tukey's HSD (Honestly Significant Difference) test since not all groups followed a normal distribution and nor did they all have equal variance.

\section{Results}

\subsection{Clinical observations}

All rats tolerated both surgeries well and returned to normal activity post-surgery. One rat from the Zol-Gel-group had to be euthanized 4 weeks after screw implantation due to a cholangiocarcinoma that was unrelated to the study. The results from this animal were still included in the analysis as the statistical tests did not identify them as outliers when compared with the other animals of the same group. The limited food intake allowed a controlled weight gain in the animals; their final mean weight was $372 \pm 21 \mathrm{~g}$.

\subsection{Assessment of the estrogen deficiency induced bone loss}

The in vivo microct scans confirmed that the rats had a diminished BMD, enlarged marrow cavities, and a significantly lower trabecular bone volume only 35 days after OVX (Table 1 ). This was shown by the decreasing BV/TV and Tb.N, and the increasing Tb.Sp. No significant changes could be detected for the Tb.Th. The rising SMI showed that the trabecular bone had shifted from a mainly plate-like structure to a more rod-like structure that is typical of osteoporotic bone [45]. However, the estrogen deficiency had no short-term influence on the cortical bone. No changes could be detected in the cortical thickness (C.Th); the TMD of the cortex increased slightly by $2 \%$.

\subsection{MicroCT-based dynamic histomorphometry}

A total of 138 microCT scans were analyzed for the dynamic histomorphometry, of which 2 had to be excluded due to motion artifacts. The sample size of the analyzed groups varied from 5 to 8 after removal of the outliers. The hyaluronic acid hydrogel used as a drug delivery system did not show any effect on the analyzed bone parameters. No significant differences were found between the Control-group and the Gel-group at any time. Volume rendered comparisons of two time points for one femur from the Gel-group and one from the Zol-Gel-group are shown in Fig. 4.

\subsubsection{Static bone parameters}

Two main bone regions can be distinguished in the results: the inner layer (0-368 $\mu \mathrm{m}$ from screw surface) and part of the second layer $(368-736 \mu \mathrm{m})$ reflect the bone reaction to the screw implantation. The two outer layers (736-1472 $\mu \mathrm{m})$ are not affected by the implant and show stable bone parameters.

All experimental groups showed a clear gain in BV/TV, Tb.Th, and Tb.N in direct proximity to the screw between day 3 and day 10 after screw implantation (Figs. 5-7). The Tb.Sp and the SMI decreased in accordance with the other bone parameters, which indicates a more compact bone structure (Figs. 8 and 9). This gain in bone mass continued for the Zol-Gel-group until day 17 and plateaued at a BV/TV of around 55\% until the end of the study. In the Control- and the Gel-groups, the initial bone gain was followed first by a rapid and then later by a moderate bone loss. The final BV/TV in those two groups was around 13\%. The effect of Zoledronate diminished with increasing distance from the screw surface. The trabecular parameters of the Zol-Gel-group showed that the gain in bone volume further from the screw was mainly caused by an increasing Tb.N and a decreasing Tb.Sp (Figs. 6 and 7). The Tb.Th in the outer two layers was identical for all groups and constant throughout the whole study (Fig. 8).

\subsubsection{Dynamic bone parameters}

In direct proximity to the screw, a constant low BRR of around $2-3 \% / d$ was seen for the Zol-Gel-group throughout the whole study (Fig. 10). The Control- and Gel-groups initially showed (days $3-10$ ) an equally low BRR followed by a high peak of around $12 \%$ / $\mathrm{d}$ from days 10 to 17 . From then onward, the BRR diminished until the study end point and reached a value equal to the Zol-Gel-group for the last period analyzed, which was 45-58 days after screw implantation. In the outer three layers, the BRR in the Control- and Gel-groups was initially very high $(8-10 \% /$ day $)$ compared to the

Table 1

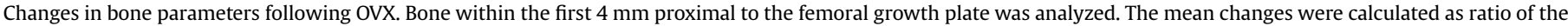
difference between the mean values before and after OVX to the mean value before OVX.

\begin{tabular}{|c|c|c|c|c|c|c|c|c|}
\hline & \multicolumn{6}{|c|}{ Cancellous bone parameters } & \multicolumn{2}{|c|}{ Cortical bone parameters } \\
\hline & BMD $\left[\mathrm{g} / \mathrm{mm}^{3}\right]$ & $\mathrm{BV} / \mathrm{TV}[\%]$ & SMI [] & Tb.Th $[\mathrm{mm}]$ & Tb.Sp [mm] & Tb.N [1/mm] & TMD $\left[\mathrm{g} / \mathrm{mm}^{3}\right]$ & C.Th $[\mathrm{mm}]$ \\
\hline Before OVX & $0.32 \pm 0.03$ & $33.4 \pm 3.9$ & $1.19 \pm 0.25$ & $0.14 \pm 0.01$ & $0.32 \pm 0.09$ & $2.39 \pm 0.32$ & $1.09 \pm 0.03$ & $0.55 \pm 0.01$ \\
\hline 35 Days after OVX & $0.23 \pm 0.04$ & $20.8 \pm 4.9$ & $1.79 \pm 0.27$ & $0.15 \pm 0.02$ & $0.92 \pm 0.25$ & $1.39 \pm 0.31$ & $1.11 \pm 0.03$ & $0.56 \pm 0.02$ \\
\hline Mean change & $-28 \%$ & $-38 \%$ & $50 \%$ & $7 \%$ & $191 \%$ & $-42 \%$ & $2 \%$ & $0 \%$ \\
\hline$p$ Value & 0.000 & 0.000 & 0.001 & 0.126 & 0.000 & 0.000 & 0.008 & 0.133 \\
\hline
\end{tabular}



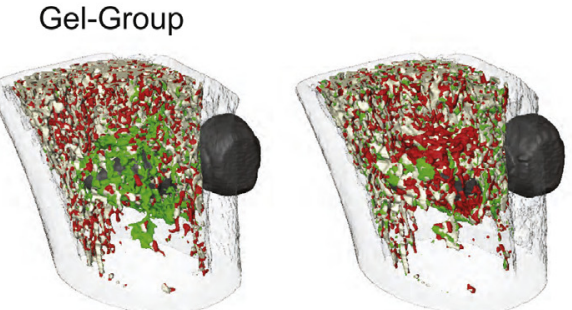

Zol-Gel-Group

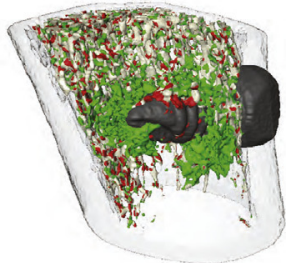

day $3-10$

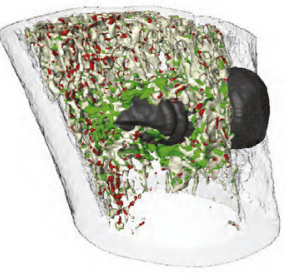

day $10-17$

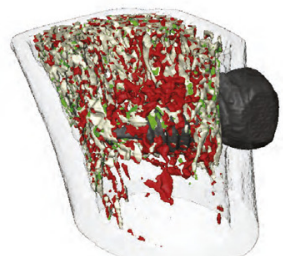

resorbed bone quiescent bone formed bone
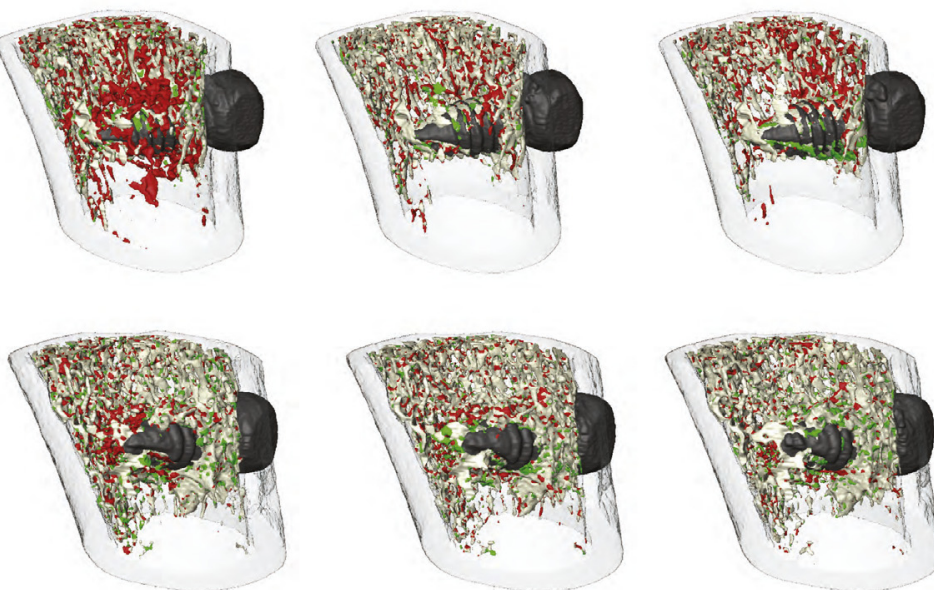

day $17-31$

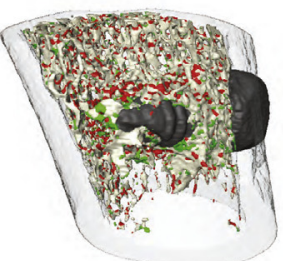

day $31-45$

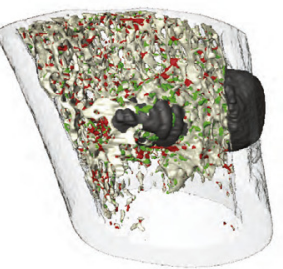

day $45-58$

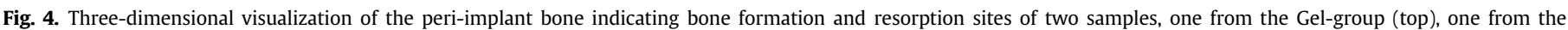

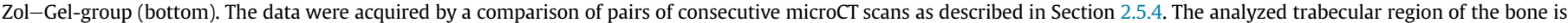
shown in light gray, red and green, the screw in dark gray and the cortex in transparent.

Zol-Gel-group (3-5\%/day). This difference evened out over time and was no longer significant from days 31 to 58 .

Unlike the effect of Zoledronate on bone resorption, its influence on bone formation was small. The results of the BFR showed that the implantation of the screw induced an early peak in bone formation in the inner two layers in all groups (Fig. 11). The BFR in the Control- and Gel-groups was characterized by a peak of around $20 \% / d$ from days 3 to 10 , followed by an almost constant low BFR of
$1-2 \% / d$ during the rest of the study. This phenomenon was also found to a lesser degree in the second layer from 368 to $736 \mu \mathrm{m}$ where the initial BFR reached $10 \% / \mathrm{d}$ and then decreased to $2-3 \% / \mathrm{d}$. The BFR in the two outer layers was unaffected by the screw implantation and showed a constant value of $3-4 \% / \mathrm{d}$. The initial peak was enhanced for the Zol-Gel-group compared to the two other groups, a significant difference was found from days 10 to 17 in the inner layer and from days 3 to 17 in the second layer. The outer two
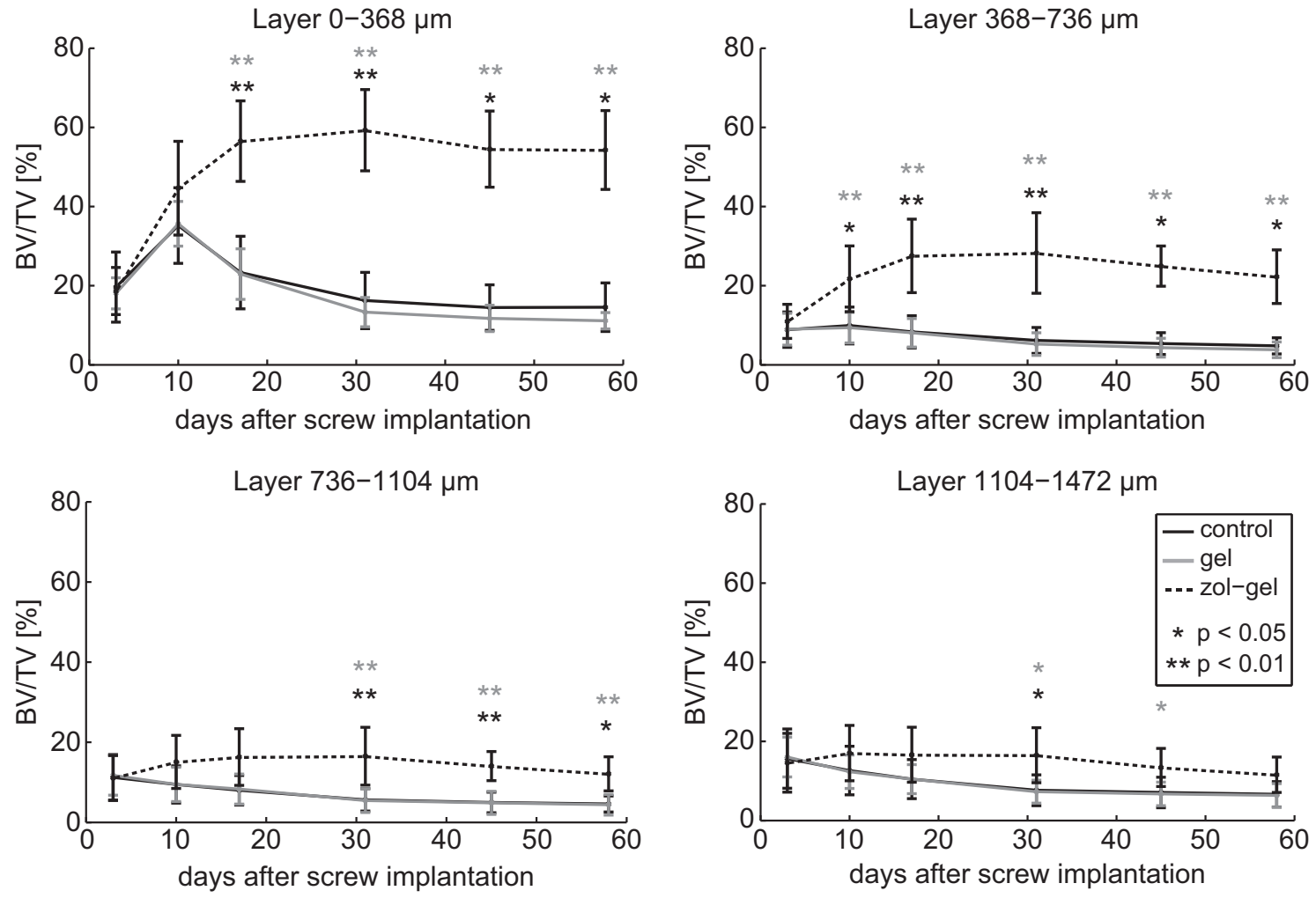

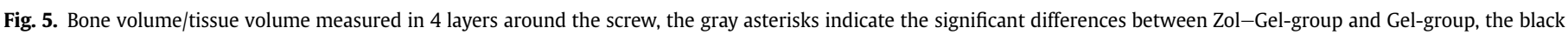
ones between Control-group and Zol-Gel-group. 

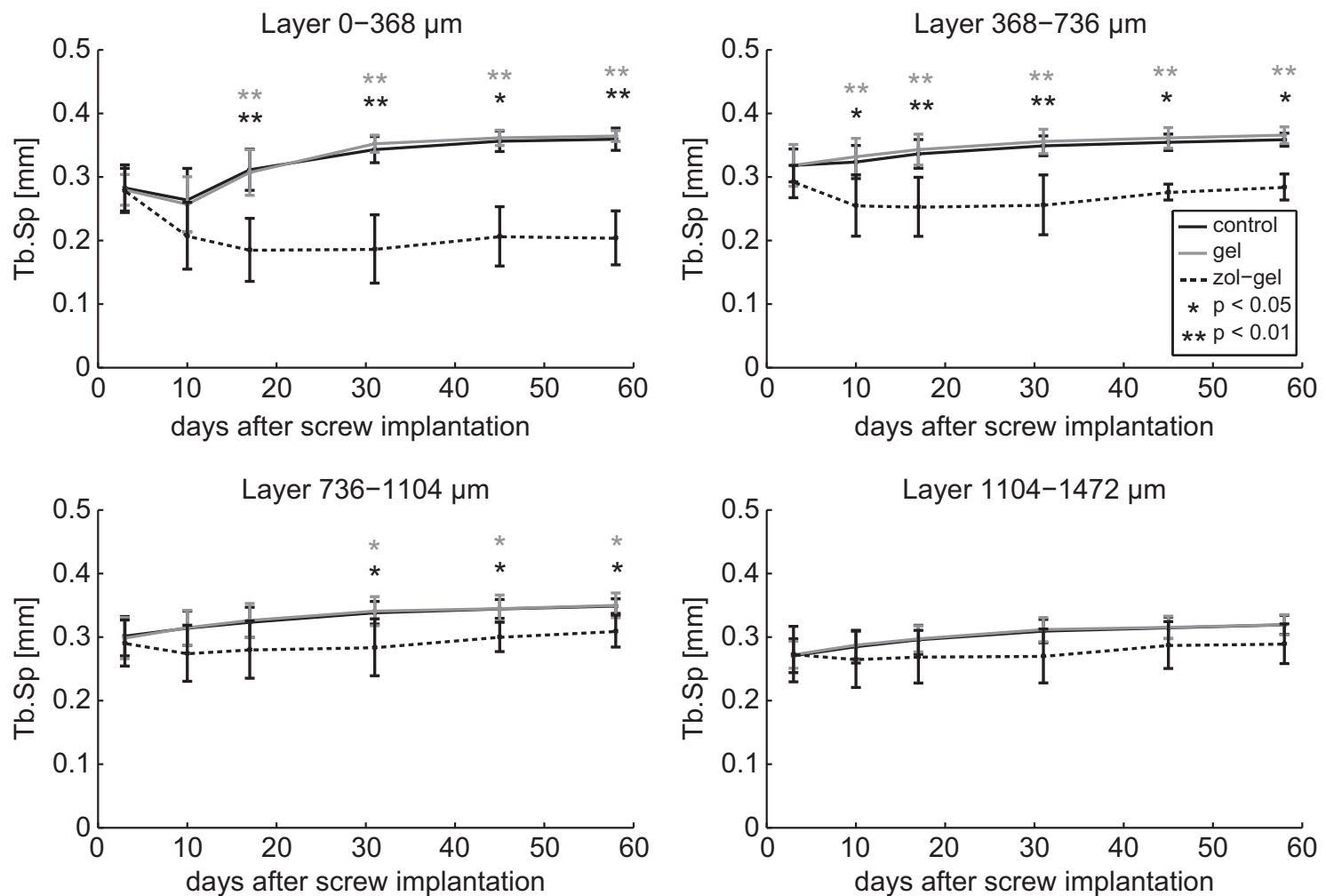

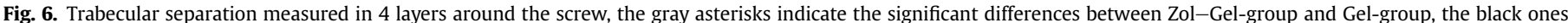
between Control-group and Zol-Gel-group.
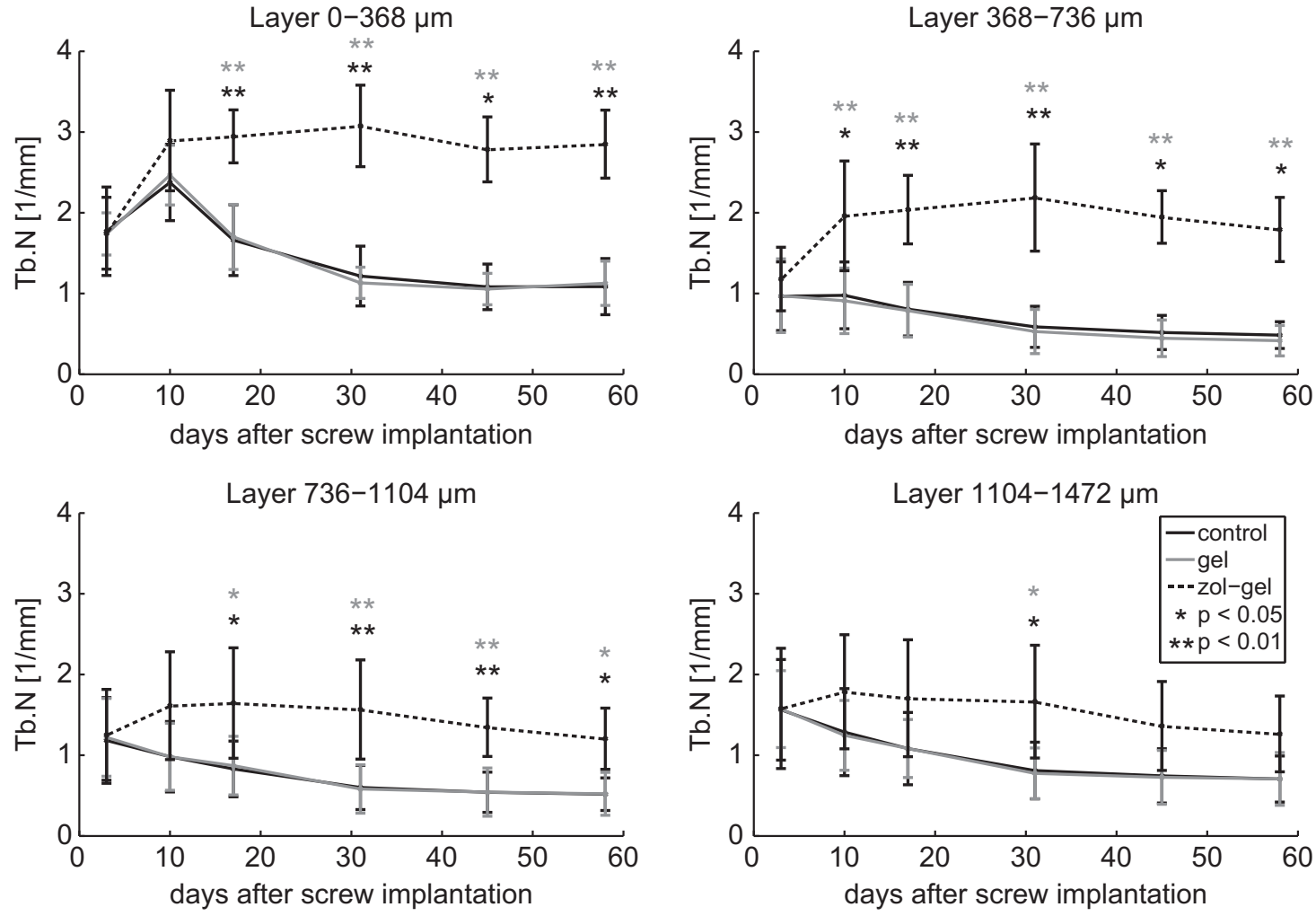

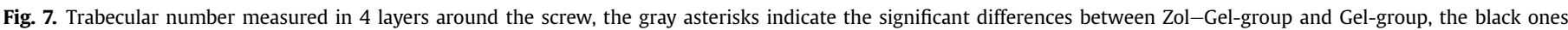
between Control-group and Zol-Gel-group. 

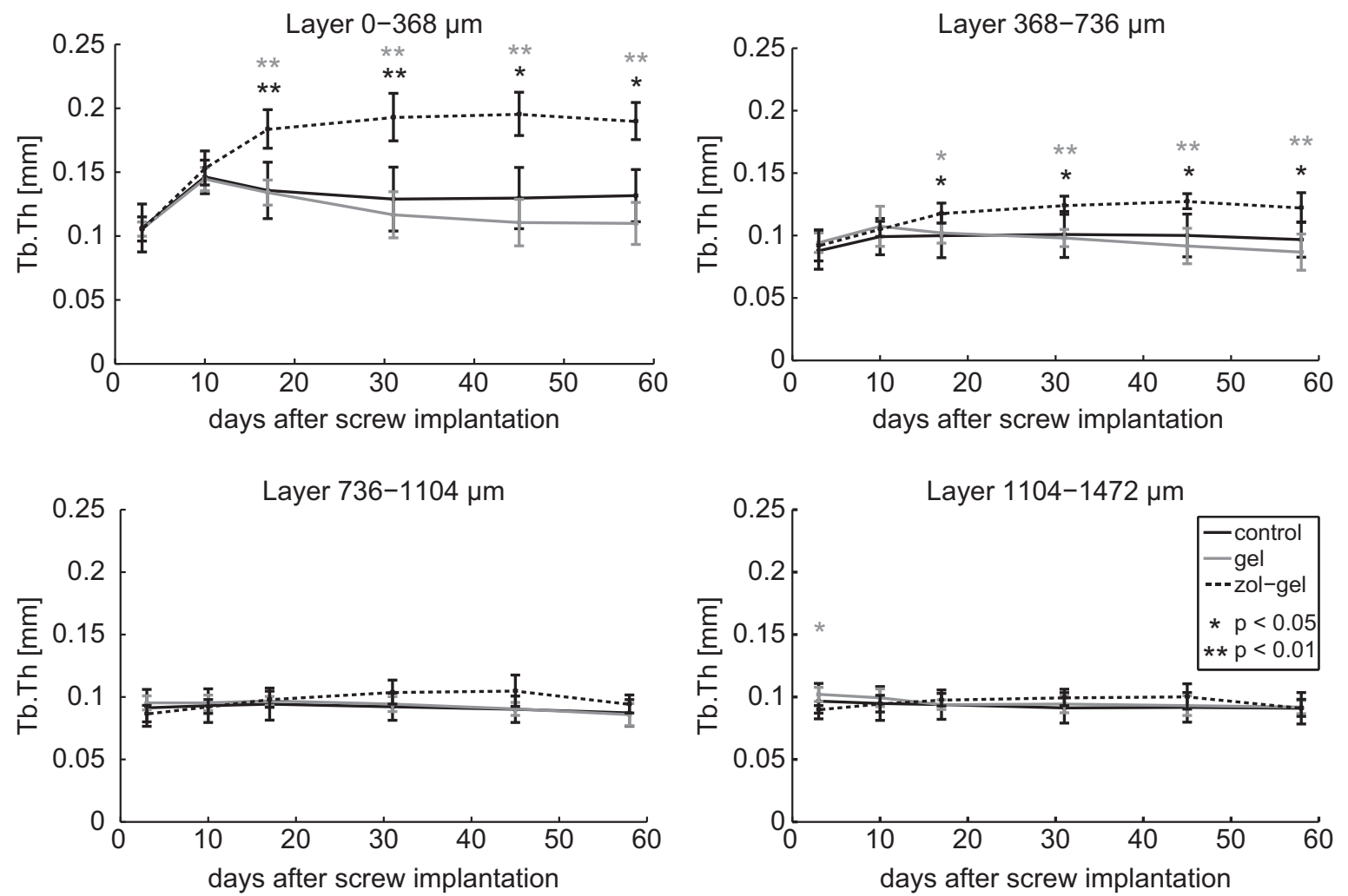

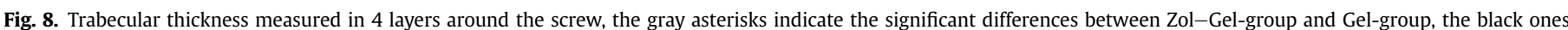
between Control-group and Zol-Gel-group.
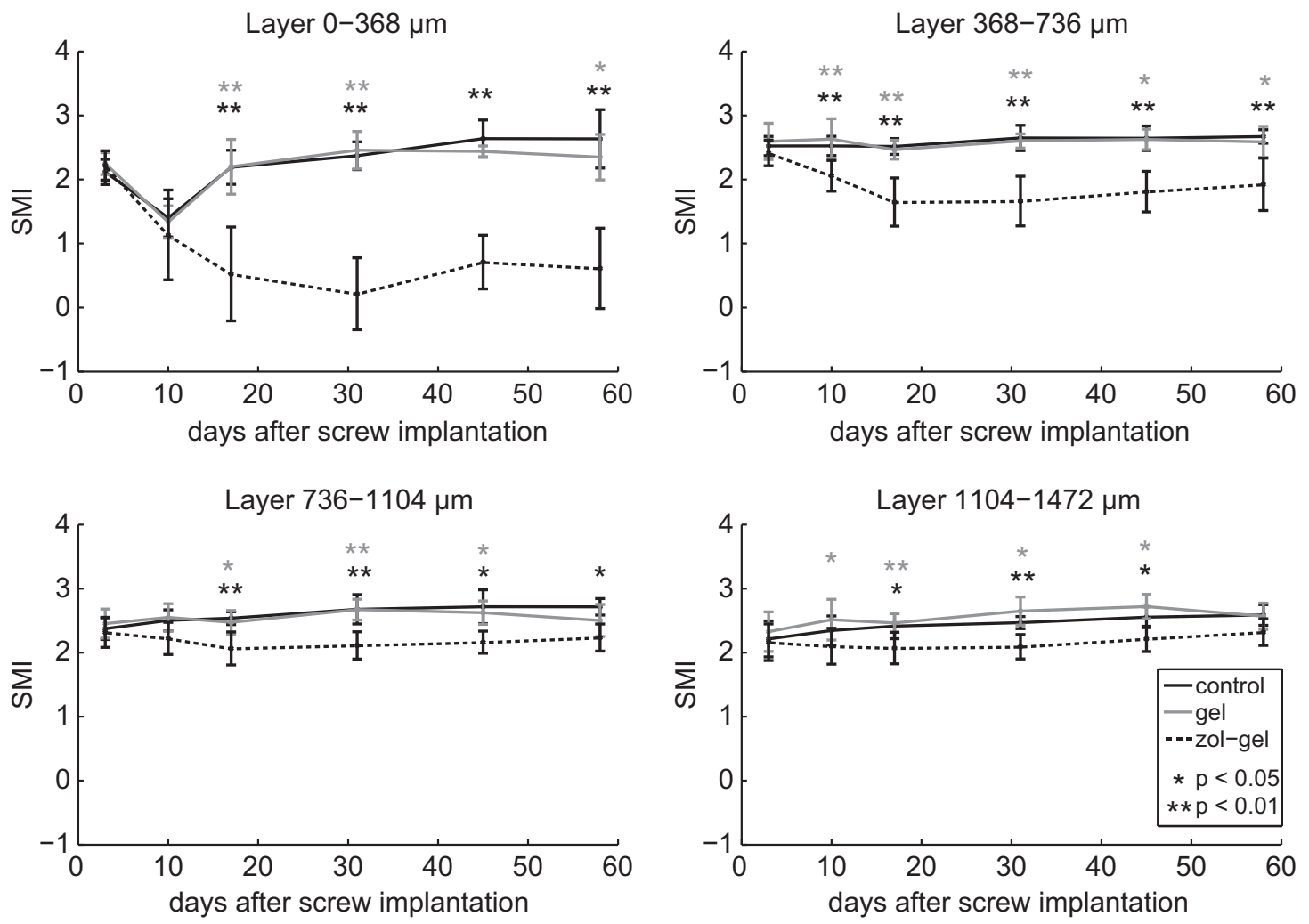

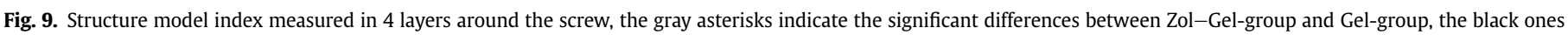
between Control-group and Zol-Gel-group. 

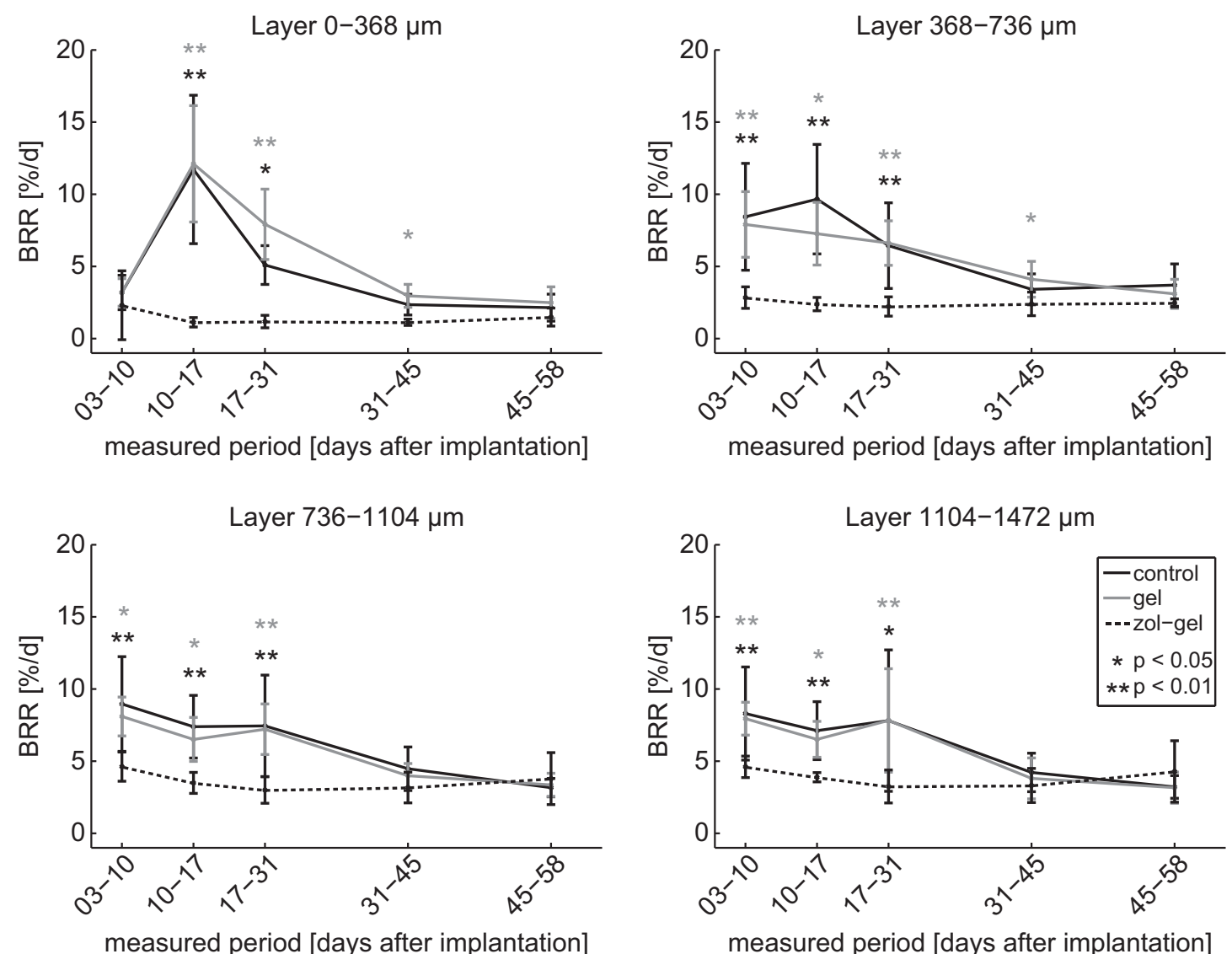

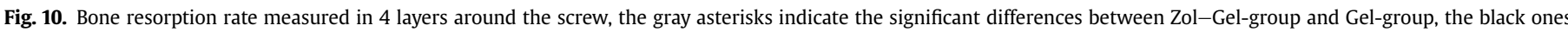
between Control-group and Zol-Gel-group.

layers of the Control- and the Gel-groups were not affected by the screw implantation and showed a constant BFR of around 5\%/d. The Zol-Gel-group differed in this region only from days 3 to 10 , where it showed a small but significant bone formation peak of $9 \% / \mathrm{d}$ in the region from 736 to $1104 \mu \mathrm{m}$ respective $6 \% / \mathrm{d}$ from 1104 to $1472 \mu \mathrm{m}$.

\subsection{Histology}

Histology results showed that the hyaluronic acid hydrogel was fully degraded after 58 days of implantation since hydrogel residues could no longer be detected in neither the Gel- nor the Zol-Gel-groups (see Fig. 12). The screws were well osteointegrated in all groups; no significant amounts of fibrotic tissue could be detected close to the screw surfaces. In the Control- and the Gelgroups, new bone matrix was deposited only in direct proximity to the screws; no new bone formation could be detected further away. Only small periosteal callus formation was visible around the screw heads of the Control- and Gel-groups, the bone structure within those calli was still immature. In contrast, a large amount of newly formed bone was detected close to and further away from the screw surface in the Zol-Gel-group. This newly formed bone also showed a woven structure typical of immature bone with an irregular arrangement of the collagen fibers, a large cell number and reduced mineral content. In the Zol-Gel-group, significant periosteal callus formation was found even far from the implantation site. Normal-shaped osteoclasts and foreign body giant cells were present in all three groups, but to a much lower extent in the Zol-Gel-group than in the other two groups. The bone lining osteoblasts seemed to be more activated in the Zol-Gel-group compared to Control- and Gel-groups. The histology showed a significantly enhanced peri-implant bone density in the Zol-Gelgroup. The newly formed bone was not fully remodeled in all groups, whereas the bone remodeling in the Zol-Gel-group seemed to be less advanced than in the other two groups.

\section{Discussion}

The main goal of the present study is to investigate the spatiotemporal effect of locally delivered Zoledronate on peri-implant bone remodeling based on time-lapsed microCT scans. In this context, a cross-linked hyaluronic acid hydrogel was used as drug delivery material.

The significant new result of this study is an up to $100 \%$ increase of the early bone formation rate caused by locally delivered Zoledronate that accompanies an efficient inhibition of periimplant bone resorption. In this way, Zoledronate initially boosts bone formation and later on helps to preserve the newly formed bone. It is the first in vivo study unambiguously demonstrating the positive effect of Zoledronate on bone formation. This result opens new possibilities for local delivery of this drug as well as for the understanding of its global effect on bone remodeling.

MicroCT-based histomorphometry was chosen for this study as it has been shown to be a very suitable technique for the quantitative analysis of three-dimensional bone formation and bone resorption rates over long in vivo time periods [36]. Obtaining data from multiple time points without having to euthanize the animals is a key advantage of a longitudinal study compared to the traditional two-dimensional histology- 

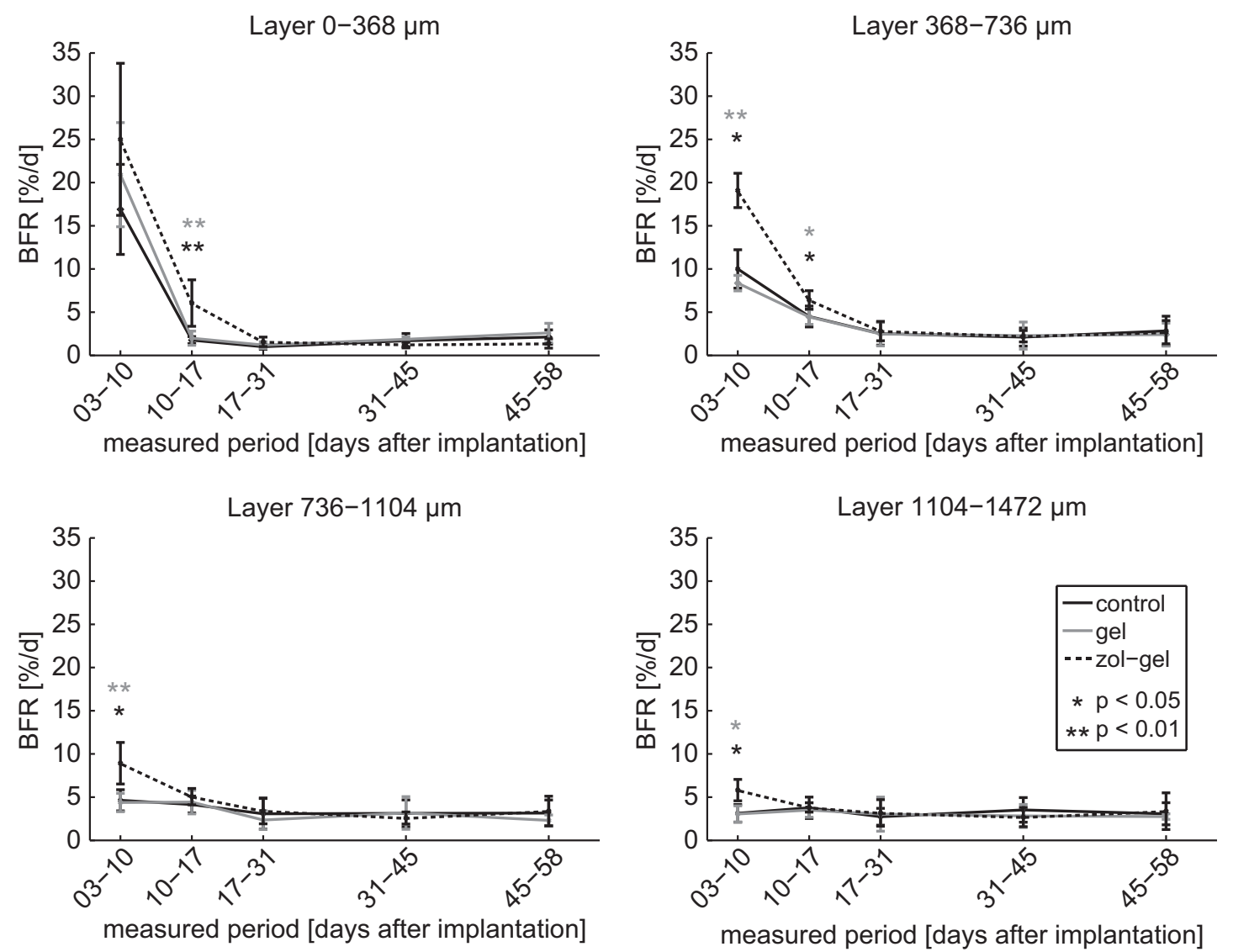

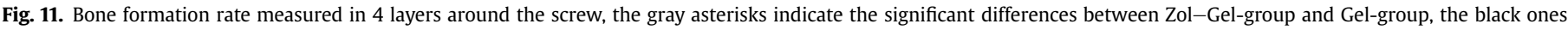
between Control-group and Zol-Gel-group.

based histomorphometry. The second benefit of the microcTbased technique compared to histology is that bone resorption rate can be quantified in a direct manner that is very useful when studying the effect of anti-resorptive drugs such as BPs [36]. Metal induced artifact issues that are typically related to CT scans and complicate the analysis of peri-implant bone were overcome in the present study by using radiopaque PEEK screws.

In order to understand the influence of the BPs on the periimplant bone remodeling, it is important to have a look at the normal bone healing as it was found in the Control-group. First, the changes in bone structure caused by the estrogen deficiency are consistent with those reported in the literature and confirm the success of the OVX [31]. The results from the histomorphometry performed after screw implantation showed that the bone reaction to the trauma caused by the screw implantation took place principally in the two innermost layers (the "bonehealing-zone", 0-736 $\mu \mathrm{m}$ from the screw surface). This zone was characterized by a large increase in bone formation rate during the first 17 days after the surgery up to a distance of $736 \mu \mathrm{m}$ from the screw surface. This early peri-implant bone formation is in accordance with literature where it was shown to be the result of the so-called static osteogenesis $[46,47]$. Osteogenic cells and a new calcified matrix are therefore deposited after only a few days on the cement line created on the implant surface [48]. This rapid deposition of calcified matrix restores the continuity of the bone structure, even if the mechanical competence of the woven bone is lower than that of mature remodeled bone due to the random orientation of the collagen fibers [49]. No microCT-based studies could be found confirming bone formation this early next to an implant.

The woven structure of the newly formed bone in the Controlgroup was confirmed in the present study by a decrease in SMI and an increase in Tb.N and Tb.Th that accompanied the gain in BV/ TV in the bone-healing-zone from days 3 to 10 . Woven bone is reported to be progressively remodeled and substituted by mature bone with a trabecular structure and delimiting marrow spaces later on [50], a process that also removes bone debris and necrotized bone caused by the implant bed preparation [51]. In the present study, the remodeling was initiated by a significant loss in bone volume in the bone-healing-zone that started 10 days after screw implantation in the Control-group.

Another interesting finding from the Control-group is the initially high, and then progressively decreasing, BRR in the outer two layers that are not in contact with the screw (the "boneremodeling-zone"). This phenomenon can be explained by the initial rapid bone loss phase induced by the estrogen deficiency of the animals that terminates 3-4 months after OVX [42]. The bone loss is even enhanced in this study by the growth of the animals that results in a "flow" of the screw and the surrounding volume of interest towards the less trabecularized diaphyseal region. Normally, the growth of rats slows down and ceases around 26 weeks of age [52], this corresponds in this study with day 31 after screw implantation. When comparing bone resorption with formation in the Control-group, it can be seen that, beside the early bone formation peak, the BRR clearly exceeded the BFR in all bone regions most of the time and leveled out only towards the end of the study. This imbalance resulted in a steady bone loss in the bone- 

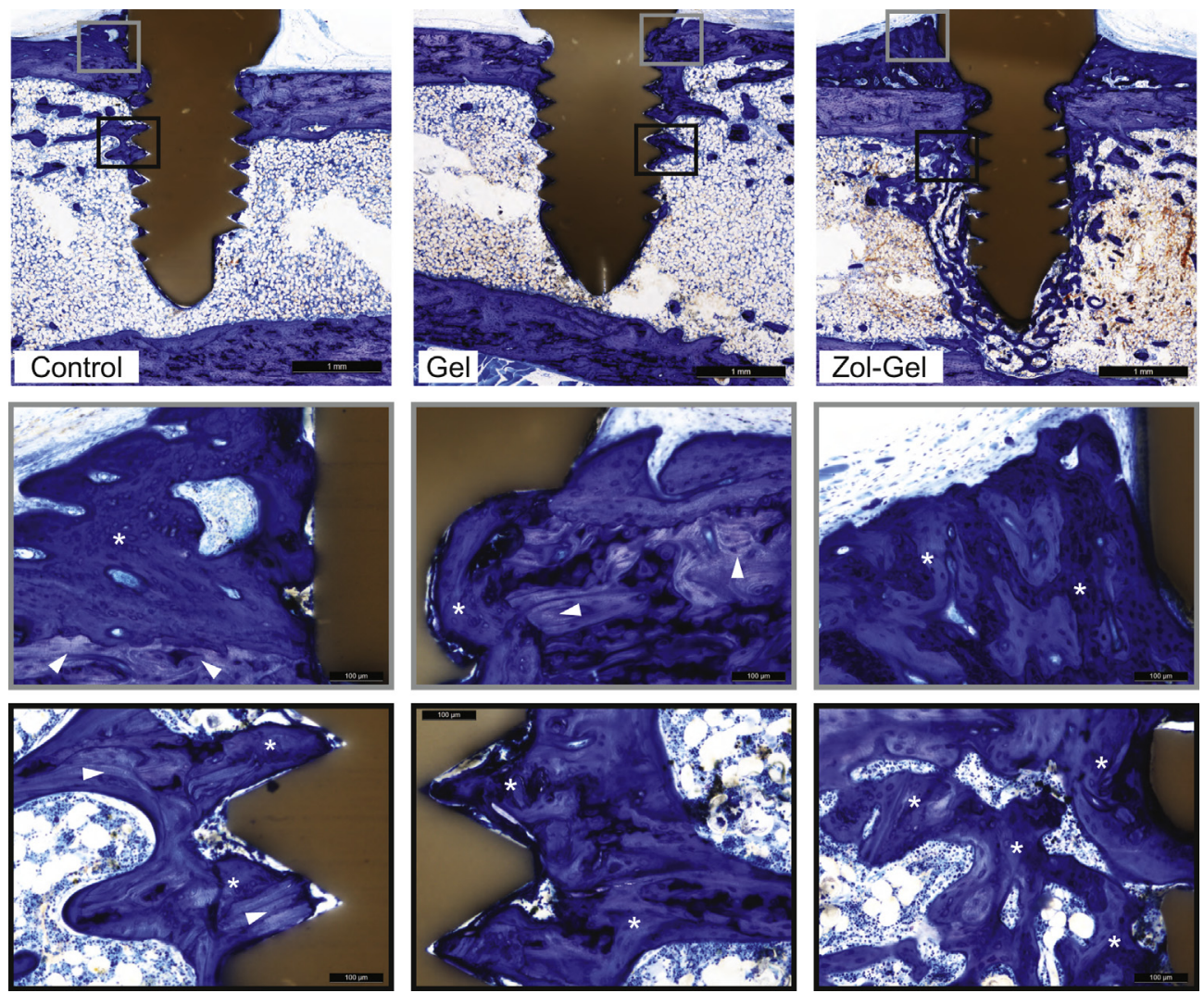

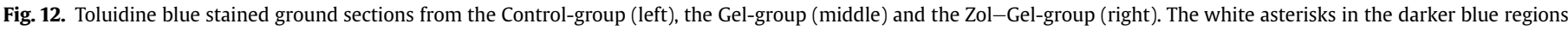

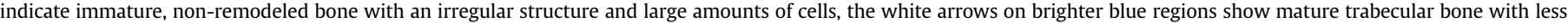
cells and a regular structure.

remodeling-zone as can be seen in the diminishing $\mathrm{BV} / \mathrm{TV}$ in the control animals.

The Gel-group did not show any statistically different results compared to the Control-group, therefore the chosen hydrogel can be considered not to have any influence on peri-implant bone remodeling. It was resorbed completely during the study without leaving any visible residuals, as confirmed by histology.

The locally delivered Zoledronate interfered with the normal healing and remodeling process at a very early stage as demonstrated in the Zol-Gel-group. An increase of up to $100 \%$ of the BFR was seen from days 3 to 17 in the bone-healing-zone and from days 3 to 10 in the bone-remodeling-zone. Later on during the study, no more differences could be detected between the groups. This result suggests that Zoledronate is only influencing bone formation during the first "flush" with the drug where bone-forming cells are either in contact with the gel or exposed to Zoledronate in solution. The direct contact could allow them to incorporate the drug via fluid phase endocytosis, an uptake mechanism for Zoledronate that has been described by Thompson et al. [53]. BPs are known to be rapidly cleared from the circulation and absorbed to bone mineral surface [54]. The fast uptake in the bone can explain why no further Zoledronate effect on bone formation could be detected later on in the study. This is because osteoclasts are the only cells that can liberate and incorporate BPs once they are bound to bone mineral [4]. It remains unclear if the initial boost in bone formation is caused by a direct stimulation of the osteoblast activity, or by unknown indirect coupling effects. Arnoldi et al. recently found a higher level of cellular proliferation and osseous differentiation at an early time point around implants coated with fibrinogen and loaded with a very low dose of Zoledronate [55]. This finding supports the theory that Zoledronate can have a direct anabolic effect on bone formation. Published in vitro studies are not conclusive as they demonstrate that Zoledronate can have positive as well as negative results on osteoblasts, depending on the dose and study design $[11,12,56]$. Orriss et al. showed that the Zoledronate dose has to be increased 10 - to 100 -fold to achieve the same inhibitory effect on osteoblasts when those are cultured on dentin slides, a finding that supports the theory that absorbed BPs are significantly less effective on osteoblasts [13].

Since Zoledronate is an anti-resorptive agent, the drug is expected to mainly influence bone resorption. In the present study, this was shown by the significantly reduced BRR in the drug treated animals. The strong resorption peak seen in the Control-group was not at all present in the bone-healing-zone of the Zol-Gel-group in which the BRR remained at a constant low level. A slightly higher but still constant BRR level could be found in the bone-remodelingzone of the Zol-Gel-group. The investigated time period was too short to detect a clear end of the drug effect on bone resorption. However, static and dynamic bone parameters of the last analyzed time points and periods suggested a diminishing drug effect with a slightly increasing BRR and decreasing differences between the groups, particularly in the bone-remodeling-zone.

Looking at the spatial effect of the locally delivered Zoledronate, this study revealed that the majority of the effects of BPs occur at a distance of up to $736 \mu \mathrm{m}$ around the screw, which corresponds to the earlier defined bone-healing-zone. Tests with hydrogel loaded with hydroxyapatite-particles (size $200 \mathrm{~nm}$ ) that give the gel a light radiopacity (data not shown) revealed that the gel is located within a range of $0-600 \mu \mathrm{m}$ around the screw. Therefore, it can be assumed that bone is in direct contact with the Zoledronate-loaded 
gel within the region that shows the strong drug effect. These results suggest that it may be possible to control the range of action of the Zoledronate-loaded gel via its penetration depth in bone. This is a promising approach as the penetration depth can be adapted by altering the mechanical properties of the gel or the volume of gel inserted into the implant bed. However, a significantly weaker drug effect was also shown for the bone-remodeling-zone that is not in contact with the gel. This finding suggests that a small part of the released Zoledronate diffuses through the bone despite its high affinity to mineral and its fast absorption in bone surfaces.

The boost in initial bone formation, the effective inhibition of the bone resorption in the bone-healing-zone, and the stabilization of the bone loss in the bone-remodeling-zone resulted in a BV/TV that was up to $300 \%$ higher compared to the Control-group in the bone-healing-zone and up to $100 \%$ higher in the bone remodelingzone. The reduction of SMI and Tb.Sp as well as the increase Tb.N confirmed a shift from osteoporotic towards a more normal bone structure. This is a very positive finding as an augmented microstructure of peri-implant bone is linked to improved implant anchorage [57-59]. The gain in bone volume and therefore in mechanical resistance might nevertheless be partly compensated for by the less mature bone structure that was present 2 months after screw implantation, as confirmed by histology in the present study. The SMI of 0.6 that characterized the bone in the bonehealing zone of the Zol-Gel-group at day 58 compared to an SMI of 2.6 in the Control-group confirmed that the very compact woven bone had not yet remodeled. Findings in similar studies provide enough evidence to show that Zoledronate treated bone can enhance implant fixation despite the reduced mechanical competence of the immature bone structure [18,19,22]. Furthermore, Amanat et al. showed with an indentation study that Zoledronate has no influence on the intrinsic mechanical properties of healing bone [60].

The present study has inherent limitations. The microcT-based dynamic histomorphometry averages bone formation and resorption rate over the time period between two scans what makes the results sensitive to the chosen time points for the scans [36]. In this study bone formation and bone resorption have to be interpreted as gain and loss of mineralized bone tissue since the microCT cannot analyze the bone on a cellular level. Furthermore, the age of the rats was not ideal due to the fact that the end of the initial OVX related bone loss phase and the cessation of bone length growth occurred during the experimental phase. In future studies, histology should be performed at early time points for a better understanding of how Zoledronate enhances very early peri-implant bone formation. It might also be useful to prolong the experimental phase in order to assess when bone maturity is reached in the Zol-Gel-group. In this study, only one drug dose of $5 \mu \mathrm{g}$ per implant was tested. A future dose-response study could help to investigate the dosedependency of the present findings.

In summary, the quickly degradable hydrogel used in this study appeared to be a very suitable drug delivery system for BPs. The texture and material properties of the gel allowed for an easy and precise application, combined with a deep penetration of the bone tissue that significantly increased the range of action of the drug. Numerous studies have shown that BPs released from coatings stay highly localized and result in a very thin layer of dense bone around implants which is not ideal for implant fixation [61,62]. This is not the case in the present study where an increased bone volume could be shown all over the analyzed trabecular region. The present study also demonstrated a positive influence of Zoledronate on early bone formation in addition to its known anti-resorptive action. This effect seems to be supported by a fast release of the small BP molecules from the highly porous hydrogel, another point in favor of the use of hydrogels as drug-delivery systems for BPs.

\section{Conclusions}

The present study was able to show that Zoledronate delivered from a quickly degrading hydrogel can boost early bone formation and later on efficiently inhibit peri-implant bone resorption close to an implant. It can also stabilize bone loss away from an implant in an OVX rat model. This process significantly enhanced bone mass and improved bone micro-structure in the treated peri-implant bone during the two months post-implantation that were studied. This is a positive finding since a reinforced bone structure is directly linked to improved implant fixation. The presented approach using Zoledronate-loaded hydrogel for implant bed preparation is highly promising, especially for patients suffering from low quality bone, though more studies are needed.

\section{Acknowledgments}

Special thanks to Sandra Jaccoud at the LBO for her assistance during surgeries and for the preparation of the histology slides. Thanks to Caroline Sieger Fernandes for the English proof-reading of the manuscript. We benefited from the help of Prof. Brigitte von Rechenberg, Dr. Karina Klein and Dr. Salim Darwiche at the University of Zurich for the interpretation of the histology slides. This study was partially supported by a KTI grant (Project no. 11098.1).

\section{References}

[1] Seeman E. Bone modeling and remodeling. Crit Rev Eukaryot Gene Expr 2009;19:219-33.

[2] Boyce BF, Rosenberg E, de Papp AE, Duong LT. The osteoclast, bone remodelling and treatment of metabolic bone disease. Eur J Clin Invest 2012;42: $1332-41$.

[3] Riggs BL, Parfitt AM. Drugs used to treat osteoporosis: the critical need for a uniform nomenclature based on their action on bone remodeling. J Bone Miner Res 2005;20:177-84.

[4] Rogers MJ, Gordon S, Benford HL, Coxon FP, Luckman SP, Monkkonen J, et al. Cellular and molecular mechanisms of action of bisphosphonates. Cancer 2000;88:2961-78.

[5] Russell RG, Watts NB, Ebetino FH, Rogers MJ. Mechanisms of action of bisphosphonates: similarities and differences and their potential influence on clinical efficacy. Osteoporos Int 2008;19:733-59.

[6] Roelofs AJ, Thompson K, Gordon S, Rogers MJ. Molecular mechanisms of action of bisphosphonates: current status. Clin Cancer Res 2006;12:6222s-30s.

[7] Idris AI, Rojas J, Greig IR, Van't Hof RJ, Ralston SH. Aminobisphosphonates cause osteoblast apoptosis and inhibit bone nodule formation in vitro. Calcif Tissue Int 2008;82:191-201.

[8] Plotkin LI, Weinstein RS, Parfitt AM, Roberson PK, Manolagas SC, Bellido T. Prevention of osteocyte and osteoblast apoptosis by bisphosphonates and calcitonin. J Clin Invest 1999;104:1363-74.

[9] Plotkin LI, Manolagas SC, Bellido T. Dissociation of the pro-apoptotic effects of bisphosphonates on osteoclasts from their anti-apoptotic effects on osteoblasts/osteocytes with novel analogs. Bone 2006;39:443-52.

[10] Giuliani N, Pedrazzoni M, Negri G, Passeri G, Impicciatore M, Girasole G. Bisphosphonates stimulate formation of osteoblast precursors and mineralized nodules in murine and human bone marrow cultures in vitro and promote early osteoblastogenesis in young and aged mice in vivo. Bone 1998;22: 455-61.

[11] Greiner S, Kadow-Romacker A, Lubberstedt M, Schmidmaier G, Wildemann B. The effect of zoledronic acid incorporated in a poly(D,L-lactide) implant coating on osteoblasts in vitro. J Biomed Mater Res A 2006;80:769-75.

[12] Pan B, To LB, Farrugia AN, Findlay DM, Green J, Gronthos S, et al. The nitrogencontaining bisphosphonate, zoledronic acid, increases mineralisation of human bone-derived cells in vitro. Bone 2004:34:112-23.

[13] Orriss IR, Key ML, Colston KW, Arnett TR. Inhibition of osteoblast function in vitro by aminobisphosphonates. J Cell Biochem 2009;106:109-18.

[14] Pazianas M, Cooper C, Ebetino FH, Russell RG. Long-term treatment with bisphosphonates and their safety in postmenopausal osteoporosis. Ther Clin Risk Manage 2010;6:325-43.

[15] Watts NB, Diab DL. Long-term use of bisphosphonates in osteoporosis. J Clin Endocrinol Metab 2010;95:1555-65.

[16] Bobyn JD, Thompson R, Lim L, Pura JA, Bobyn K, Tanzer M. Local alendronic acid elution increases net periimplant bone formation: a micro-CT analysis, Clin Orthop Relat Res 2014;472:687-94. 
[17] Roshan-Ghias A, Arnoldi J, Procter P, Pioletti DP. In vivo assessment of local effects after application of bone screws delivering bisphosphonates into a compromised cancellous bone site. Clin Biomech 2011;26:1039-43.

[18] Peter B, Gauthier O, Laib S, Bujoli B, Guicheux J, Janvier P, et al. Local delivery of bisphosphonate from coated orthopedic implants increases implants mechanical stability in osteoporotic rats. J Biomed Mater Res A 2006;76: $133-43$.

[19] Tengvall P, Skoglund B, Askendal A, Aspenberg P. Surface immobilized bisphosphonate improves stainless-steel screw fixation in rats. Biomaterials 2004;25:2133-8.

[20] Astrand J, Aspenberg P. Topical, single dose bisphosphonate treatment reduced bone resorption in a rat model for prosthetic loosening. J Orthop Res 2004;22:244-9.

[21] Hilding M, Aspenberg P. Local peroperative treatment with a bisphosphonate improves the fixation of total knee prostheses: a randomized, double-blind radiostereometric study of 50 patients. Acta Orthop 2007;78:795-9.

[22] Andersson T, Agholme F, Aspenberg P, Tengvall P. Surface immobilized zoledronate improves screw fixation in rat bone: a new method for the coating of metal implants. J Mater Sci Mater Med 2010;21:3029-37.

[23] Cottrell JA, Vales FM, Schachter D, Wadsworth S, Gundlapalli R, Kapadia R, et al. Osteogenic activity of locally applied small molecule drugs in a rat femur defect model. J Biomed Biotechnol 2010;2010:597641.

[24] Srisubut S, Teerakapong A, Vattraphodes T, Taweechaisupapong S. Effect of local delivery of alendronate on bone formation in bioactive glass grafting in rats. Oral Surg Oral Med Oral Pathol Oral Radiol Endodontol 2007;104:e11-6.

[25] Åstrand J, Harding AK, Aspenberg P, Tägil M. Systemic zoledronate treatment both prevents resorption of allograft bone and increases the retention of new formed bone during revascularization and remodelling. A bone chamber study in rats. BMC Musculoskelet Disord 2006:7.

[26] Jakobsen T, Baas J, Bechtold JE, Elmengaard B, Soballe K. The effect of soaking allograft in bisphosphonate: a pilot dose-response study. Clin Orthop Relat Res 2010;468:867-74.

[27] Cheng TL, Murphy CM, Cantrill LC, Mikulec K, Carpenter C, Schindeler A, et al. Local delivery of recombinant human bone morphogenetic proteins and bisphosphonate via sucrose acetate isobutyrate can prevent femoral head collapse in Legg-Calve-Perthes disease: a pilot study in pigs. Int Orthop 2013: $1-7$.

[28] Back DA, Pauly S, Rommel L, Haas NP, Schmidmaier G, Wildemann B, et al. Effect of local zoledronate on implant osseointegration in a rat model. BMC Musculoskelet Disord 2012:13.

[29] Recker RR, Delmas PD, Halse J, Reid IR, Boonen S, Garcia-Hernandez PA, et al. Effects of intravenous zoledronic acid once yearly on bone remodeling and bone structure. J Bone Min Res 2008;23:6-16.

[30] Gasser JA, Ingold P, Venturiere A, Shen V, Green JR. Long-term protective effects of zoledronic acid on cancellous and cortical bone in the ovariectomized rat. J Bone Min Res 2008;23:544-51.

[31] Brouwers JEM, Lambers FM, Gasser JA, Van Rietbergen B, Huiskes R. Bone degeneration and recovery after early and late bisphosphonate treatment of ovariectomized Wistar rats assessed by in vivo micro-computed tomography. Calcif Tissue Int 2008;82:202-11.

[32] Fuchs RK, Phipps RJ, Burr DB. Recovery of trabecular and cortical bone turnover after discontinuation of risedronate and alendronate therapy in ovariectomized rats. J Bone Min Res 2008;23:1689-97.

[33] Tobias JH, Chow JWM, Chambers TJ. 3-Amino-1-hydroxypropylidine-1bisphosphonate (AHPrBP) suppresses not only the induction of new, but also the persistence of existing bone-forming surfaces in rat cancellous bone. Bone 1993;14:619-23.

[34] Belfrage O, Isaksson $\mathrm{H}$, Tägil $\mathrm{M}$. Local treatment of a bone graft by soaking in zoledronic acid inhibits bone resorption and bone formation. A bone chamber study in rats. BMC Musculoskelet Disord 2012:13.

[35] Allen MR, Follet H, Khurana M, Sato M, Burr DB. Antiremodeling agents influence osteoblast activity differently in modeling and remodeling sites of canine rib. Calcif Tissue Int 2006;79:255-61.

[36] Schulte FA, Lambers FM, Kuhn G, Muller R. In vivo micro-computed tomography allows direct three-dimensional quantification of both bone formation and bone resorption parameters using time-lapsed imaging. Bone 2011;48: 433-42.

[37] Waarsing JH, Day JS, Van Der Linden JC, Ederveen AG, Spanjers C, De Clerck N, et al. Detecting and tracking local changes in the tibiae of individual rats: a novel method to analyse longitudinal in vivo micro-CT data. Bone 2004;34: 163-9.
[38] Bergman K, Engstrand T, Hilborn J, Ossipov D, Piskounova S, Bowden T. Injectable cell-free template for bone-tissue formation. J Biomed Mater Res Part A 2009:91:1111-8.

[39] Kisiel M, Klar AS, Martino MM, Ventura M, Hilborn J. Evaluation of injectable constructs for bone repair with a subperiosteal cranial model in the rat. PLoS One 2013:8.

[40] Martínez-Álvarez C, González-Meli B, Berenguer-Froehner B, Paradas-Lara I López-Gordillo Y, Rodríguez-Bobada C, et al. Injection and adhesion palatoplasty: a preliminary study in a canine model. J Surg Res 2013;183:654-62.

[41] Martínez-Sanz E, Varghese OP, Kisiel M, Engstrand T, Reich KM, Bohner M, et al. Minimally invasive mandibular bone augmentation using injectable hydrogels. J Tissue Eng Regen Med 2012;6:s15-23.

[42] Hogan HA, Ruhmann SP, Sampson HW. The mechanical properties of cancellous bone in the proximal tibia of ovariectomized rats. J Bone Min Res 2000;15:284-92.

43] Alghamdi HS, Bosco R, van den Beucken JJP, Walboomers XF, Jansen JA Osteogenicity of titanium implants coated with calcium phosphate or collagen type-I in osteoporotic rats. Biomaterials 2013;34:3747-57.

[44] Brouwers JEM, Van Rietbergen B, Huiskes R. No effects of in vivo micro-CT radiation on structural parameters and bone marrow cells in proximal tibia of Wistar rats detected after eight weekly scans. J Orthop Res 2007;25: 1325-32.

[45] Hildebrand T, Rüegsegger P. Quantification of bone microarchitecture with the structure model index. Comput Methods Biomech Biomed Eng 1997:1: $15-23$.

[46] Marco F, Milena F, Gianluca G, Vittoria O. Peri-implant osteogenesis in health and osteoporosis. Micron 2005:36:630-44.

[47] Ferretti M, Palumbo C, Contri M, Marotti G. Static and dynamic osteogenesis: two different types of bone formation. Anat Embryol (Berl) 2002;206:21-9.

[48] Meyer U, Joos U, Mythili J, Stamm T, Hohoff A, Fillies T, et al. Ultrastructura characterization of the implant/bone interface of immediately loaded dental implants. Biomaterials 2004;25:1959-67.

[49] Probst A, Spiegel HU. Cellular mechanisms of bone repair. J Invest Surg 1997:10:77-86

[50] Franchi M, Orsini E, Trire A, Quaranta M, Martini D, Piccari GG, et al. Osteogenesis and morphology of the peri-implant bone facing dental implants ScientificWorldJournal 2004:4:1083-95.

[51] Futami T, Fujii N, Ohnishi H, Taguchi N, Kusakari H, Ohshima H, et al. Tissue response to titanium implants in the rat maxilla: ultra structural and histochemical observations of the bone-titanium interface. J Periodontol 2000;71: 287-98.

[52] Walker KV, Kember NF. Cell kinetics of growth cartilage in the rat tibia. II. Measurements during ageing. Cell Tissue Kinet 1972;5:409-19.

[53] Thompson K, Rogers MJ, Coxon FP, Crockett JC. Cytosolic entry of bisphosphonate drugs requires acidification of vesicles after fluid-phase endocytosis. Mol Pharmacol 2006;69:1624-32.

[54] Lin JH. Bisphosphonates: a review of their pharmacokinetic properties. Bone 1996;18:75-85.

[55] Arnoldi J, Alves A, Procter P. Early tissue responses to zoledronate, locally delivered by bone screw, into a compromised cancellous bone site: a pilot study. BMC Musculoskelet Disord 2014:15.

[56] Walter C, Klein MO, Pabst A, Al-Nawas B, Duschner H, Ziebart T. Influence of bisphosphonates on endothelial cells, fibroblasts, and osteogenic cells. Clin Oral Investig 2010;14:35-41.

[57] Wirth AJ, Goldhahn J, Flaig C, Arbenz P, Müller R, Van Lenthe GH. Implant stability is affected by local bone microstructural quality. Bone 2011;49:473-8.

[58] Wirth AJ, Müller R, Van Lenthe GH. Augmentation of peri-implant bone improves implant stability: quantification using simulated bone loss. J Orthop Res 2012;30:178-84.

[59] Yakacki CM, Poukalova M, Guldberg RE, Lin A, Saing M, Gillogly S, et al. The effect of the trabecular microstructure on the pullout strength of suture anchors. J Biomech 2010;43:1953-9.

[60] Amanat N, He LH, Swain MV, Little DG. The effect of zoledronic acid on the intrinsic material properties of healing bone: an indentation study. Med Eng Phys 2008;30:843-7.

[61] McKenzie K, Dennis Bobyn J, Roberts J, Karabasz D, Tanzer M. Bisphosphonate remains highly localized after elution from porous implants. Clin Orthop Relat Res 2011;469:514-22.

[62] Stadelmann VA, Gauthier O, Terrier A, Bouler JM, Pioletti DP. Implants delivering bisphosphonate locally increase periprosthetic bone density in an osteoporotic sheep model. A pilot study. Eur Cell Mater 2008;16:10-6. 